AperTO - Archivio Istituzionale Open Access dell'Università di Torino

\title{
Feedback Systems: An Analytical Framework
}

\section{This is the author's manuscript}

Original Citation:

Availability:

This version is available http://hdl.handle.net/2318/142348

since 2016-06-14T12:53:47Z

Published version:

DOI:10.1162/COMJ a 00176

Terms of use:

Open Access

Anyone can freely access the full text of works made available as "Open Access". Works made available under a Creative Commons license can be used according to the terms and conditions of said license. Use of all other works requires consent of the right holder (author or publisher) if not exempted from copyright protection by the applicable law. 


\section{Dario Sanfilippo* and Andrea Valle ${ }^{\dagger}$}

*Scuola di Musica Elettronica

Conservatorio di Musica San Pietro a

Majella di Napoli

Via San Pietro a Majella, 35

80133 Napoli, Italy

sanfilippo.dario@gmail.com

${ }^{\dagger}$ Centro Interdipartimentale di Ricerca su

Multimedia e Audiovisivo (CIRMA)/

Dipartimento di Studi Umanistici

Università degli Studi di Torino

via Sant'Ottavio 20

10124 Torino, Italy

andrea.valle@unito.it

\section{Feedback Systems: An Analytical Framework}

\begin{abstract}
The use of feedback-based systems in the music domain dates back to the 1960s. Their applications span from music composition and sound organization to audio synthesis and processing, as the interest in feedback resulted both from theoretical reflection on cybernetics and system theory, and from practical experimentation on analog circuits. The advent of computers has made possible the implementation of complex theoretical systems in audio-domain oriented applications, in some sense bridging the gap between theory and practice in the analog domain, and further increasing the range of audio and musical applications of feedback systems.

In this article we first sketch a minimal history of feedback in music; second, we briefly introduce feedback systems from a theoretical point of view; then we propose a set of features that characterize them from the perspective of music applications; finally, we propose a typology targeted at feedback systems used in the audio/musical domain and discuss some relevant examples.
\end{abstract}

\section{Some Brief Historical Remarks}

The use of feedback systems gained popular momentum in the 1960s in relation to the success of cybernetics (Wiener 1948) and system theory (von Bertalanffy 1968), two trans-disciplinary epistemological approaches that, starting from the 1940s, strongly emphasized the relevance of closed information loops in organized structures. Nicolas Schöffer (1954) was the first in art history to advocate the use of cybernetic systems in his "spatio-dynamic" works. In 1955 he created the first physical installation implementing a self-regulating mechanism, the CYSP 1. Installed in Paris, this cybernetic sculpture included some computational capabilities thanks to the technology offered by the Philips company. Provided with photocells and a microphone, CYSP 1 was capable of sensing the environment-including itself-and of sonically reacting by playing back sounds composed by French composer Pierre Henry (Prieberg 1960).

Computer Music Journal, 37:2, pp. 12-27, Summer 2013 doi:10.1162/COMJ_a_00176

(c) 2013 Massachusetts Institute of Technology.
Another seminal experience, although scarcely recognized, was Roland Kayn's musical output from 1964. Drawing inspiration from cybernetics, the German composer experimented extensively with auto-regulating systems based on feedback loops, both as formal models for instrumental composition and as networks of analog signal generators for electronic music (Patteson 2012).

During the same decade a second suggestion came from signal theory and acoustics, through the investigation of the Larsen phenomenon discovered some decades earlier. The Larsen effect happens when-given sufficient amplification-the sound captured from a microphone connected to a speaker is reproduced and again captured, recursively, resulting in a positive feedback that produces pitched tones from the iterated amplification of a signal (Boner and Boner 1966). Since then, feedback in the audio domain has been extensively exploited by pop and rock musicians, in particular guitar players incorporating feedback in their style as a result of the interaction between the two poles of the electroacoustic chain, pickups on the one hand and, on the other, amplifiers and loudspeakers, with intermediate effects playing a fundamental role. The first case is probably I Feel Fine by The 
Beatles in 1964, and the most significant example is Jimi Hendrix intensively exploring feedback since the first album with his band The Jimi Hendrix Experience (Are You Experienced from 1967, e.g., the opening of Foxy Lady, see Hodgson 2010, p. 118ff.). Furthermore, the empirical practices emerging in the 1960s from hardware hacking and circuit bending have also contributed to the development and spreading of that approach, considering that they can be intrinsically feedback-related.

Reed Ghazala was one of the pioneers of circuit bending, a practice that, even if in a nonsystematic way, includes structural feedback as a possible outcome of empirical hacking with jumper wires on existing circuits (Ghazala 2005).

Other musicians have probably discovered feedback in music simply by experimenting with their equipment, without necessarily having a complete awareness of what was occurring, while also being able to understand the charm and potential of this phenomenon. Among the first composers to incorporate feedback in their work were Robert Ashley (The Wolfman, 1964), John Cage (Electronic Music for Piano, 1964), Steve Reich (Pendulum Music, 1968), and Alvin Lucier (I Am Sitting in a Room, 1969), as well as Gordon Mumma (Hornpipe, 1967) who worked extensively with self-constructed circuits, and David Tudor (Tone Burst, 1975), who was particularly relevant, basing many of his practices exclusively on feedback (Microphone, 1973). This interest was also common among video artists, many of whom who were analogously experimenting in the same years with optical feedback (in particular Nam June Paik; Steina and Woody Vasulka; and Bill Viola), and the interest originates in both cases in the possibility of experimenting with recording technologies. The case of Bill Viola is particularly interesting: his work Information (1973) stems from a technical mistake, in which a videotape recorder tried to record its output, with Viola intervening in the loop by means of a switch (Viola 1995). As Viola himself recalls, in his formative years he was associated with David Tudor and deeply exposed to Tudor's works and thinking (Viola 2004).

Computer-based technologies have made a wider set of possibilities in terms of control over sound generation and organization available to both composers and performers. Not by chance, many composers, performers, and sound artists now work intensively with computer-based feedback systems. As an example, to name but a few from the Italian scene, and apart from Agostino Di Scipio, whom we discuss subsequently: Marco Cecotto, with his Inner Voices-A Conversation (2013), an audiovisual installation based on automated processing of Larsen tones (http://vimeo.com/62904221); Roberto Pugliese, who in 2011 created, in his Equilibrium Variant, a pair of mechanical arms interacting by listening to and producing Larsen effects (www.robertopugliese.com/page2/page22/page22 .html); Simone Pappalardo, who, through electromagnetic emitters and receivers, creates electromagnetic feedback loops that eventually turn into music systems for hybrid performance/installation works, like his Room 3327 (www.simone-pappalardo .it/Simone-Pappalardo/room3327.html); and Massimo Scamarcio, who experimented with an audio ecosystem populated by resonant filters in feedback (Scamarcio 2008).

In recent decades, feedback has also played a major technological role in digital signal processing. A feedback oscillator instrument first appeared in JeanClaude Risset's Introductory Catalog of Computer Generated Sounds in 1969. Another example is the self-modulating oscillator using amplitude modulation (where the output signal is used to control the modulation index) that was implemented in the Music V language. Subsequently, the technique (this time with feedback frequency modulation) gained wide popularity because of Yamaha's patented application of the method in its digital synthesizers (Roads 1996). Feedback has made it possible to model filters whose behavior is representative of analog filters (infinite impulse response filters), which under specific conditions turn into sound generators such as the digital resonator, a second-order bandpass filter where stable self-oscillation occurs and allows the implementation of sample-quality sinusoidal oscillators. The notion of a recirculating wavetable in the well-known Karplus-Strong algorithm for plucked-string and drum synthesis is based on a noise source initially populating a table that is then put through a feedback mechanism that re-populates the same table with the output samples after they have been processed by a modifier element (Karplus and Strong 1983).

Sanfilippo and Valle 
In general, feedback is particularly good for modeling acoustic interactions, and, not by chance, physical model implementations and digital reverberators usually include a feedback mechanism as a crucial element (see, e.g., Cook 1992; Rocchesso and Smith 1997). In the rest of this article, we will focus on the musical application of feedback systems, even if most considerations apply to feedback in general.

\section{Feedback Properties}

A minimal definition of feedback takes into account the configuration of a system, provided with input and output, in which some kind of transformation occurs, where the output is connected (fed back) to the input after a delay (de Rosnay 1997). From a theoretical point of view, we can think of a zerodelay feedback loop as a system whose fundamental frequency is infinity; in practical terms, any implementation and performance of a feedback system implies a delay greater than zero.

In negative feedback the input-output relation is inverse: An increase in the output causes a decrease in the input, and vice versa. Thus, the response of the system to stimuli is that of compensation, and it will tend to be in equilibrium around a desired target. In a positive feedback configuration, the input-output relation is direct: If the output increases, the input increases; and, vice versa, if the output decreases, the input decreases. In this case, a deviation of the system in a certain direction will produce a further shifting in the same direction, and the behavior will be that of magnifying the effects caused by the stimuli (Wiener 1948; Ashby 1956; Heylighen 2001; Heylighen and Joslyn 2001; Gershenson 2007).

As discussed by Heylighen and Joslyn (2001), the positive and negative feedback concepts can also be generalized as causal relations. In a system with a causal relation between two variables $A \rightarrow B$, a positive feedback occurs if an increase (or decrease) in $A$ produces a corresponding increase (or decrease) in $B$; likewise, a negative feedback occurs when an increase (or decrease) in $A$ produces a decrease (or increase) in $B$. For example, in the relation infected people $\rightarrow$ viruses, an increase in the infected people will lead to an increase in viruses, which will in turn lead to an increase in infected people (positive feedback). In the relation rabbits $\rightarrow$ grass, more rabbits eat more grass, grass decreases and so will the rabbit population, but a decrease in the rabbit population allows more grass to grow, eventually leading to more rabbits, and so on (negative feedback). Negative feedback is widely used in self-regulating and other control systems (from thermostats to living organisms), and its major role is that of creating stability. Positive feedback, instead, is typically an unstable behavior and will lead to exponential variations.

A set of specific features emerges in the literature on the general subject of feedback systems, but there also exists much specific to the audio/musical domain. As a consequence, a specific corpus of works and practices that share these features can be identified in electronic and electroacoustic music. Even if this specific body of work can be identified through the use of these features, there is a great variety within this body of work, and a classification schema can be proposed in order to clarify how systems differ. In the following we first introduce the set of features that can be recognized in feedback systems, then we propose a classification schema for the identified works.

\section{Nonlinearity and Circular Causality}

A system is said to be linear when its output (effects) is proportional to its input (causes). As an example, let us consider a billiard ball not subject to friction forces. If the ball is hit with a force $f$, it will have a velocity $v$. When the force is twice greater, the velocity is doubled. Actually, many natural phenomena and systems in the world are intrinsically nonlinear, having no proportional relation between causes and effects. As a result, in a nonlinear system, causes of reduced size can have greater effects, and, on the other hand, causes of greater size can have smaller effects. A feedback system is typically nonlinear, the nonlinearity being the result of a process with circular causality (Heylighen 2001; Gershenson 2007). In such a configuration, effects are also causes (Heylighen 2001), and there is a mutual relation between them. 
The causes are fed back to themselves through their effects, and the effects are the result of their combination with the causes, thus breaking the input-output linear proportion.

Another important feature of feedback configuration and circular causality is that processes become iterated, leading to systems which will be capable of self-alimentation. From a musical perspective, nonlinearity clearly emerges in feedback-based systems where the change of internal variables can result in very different behaviors in the final output. A clear example is provided by the work of Japanese improviser Toshimaru Nakamura. Talking about his performance with the No-Input Mixing Board system (see subsequent discussion), he states in a YouTube video ("No-input, Sachiko M and Toshimaru Nakamura," www.youtube.com/watch?v=Tl8IMc-8-N8, starting at $\left.5^{\prime} 05^{\prime \prime}\right)$ :

You can't totally control no-input music because it's all about feedback. Things like turning the tuning knob, even by one millimeter, make a big difference to the sound. ... It's very hard to control it. The slightest thing can change the sound. It's unpredictable and uncontrollable. Which makes it challenging. But, in a sense, it's because of the challenges that I play it. I'm not interested in playing music that has no risk.

It is not surprising to realize that different kinds of sonic features (amplitude, pitch, sustain, spectral roughness, etc.) that can be considered substantially unrelated in linear audio systems are instead deeply interrelated in feedback configurations, where a modification of one sonic feature can potentially lead to modifications in all the others.

\section{Interaction, Interdependency, and Synergy}

A fundamental property in feedback configurations is that of coupling (Ashby 1956). Two or more elements within a feedback loop are coupled because they operate in a condition where they mutually affect each other. From a systemic perspective the concepts of interaction, interdependency, and synergy are crucial to understanding feedback systems. A totality which is made up of different components, interconnected by specific relations, shows a certain behavior and identity, thanks to the cooperation of all its parts. Any small change in the organization of the relational network can potentially change the identity of the system and radically alter its behavior. Any system of this type, therefore, relies on all its components, and each of the parts has a fundamental role in the global functioning of the system. The strict interaction between the components allows for the combination of their properties, leading to new entities that are not the result of a mere summation of the properties of their parts, but rather the result of their synergy.

In most cases, so-called interaction in performances is described as a high-level relation occurring between the human and the machine, where-typically-gestural devices let the performer define actions to be followed by reactions in the machine, without taking into account a real mutual influence. In contrast, Di Scipio (2003) has been able to provide an interesting perspective on interaction in music by describing it as a condition that takes place in the sound domain: Interaction occurs among sound materials.

Christopher Burns followed Di Scipio's path in his realization of Electronic Music for Piano by John Cage (Burns 2004). He implemented a system based on a network of eight delay lines in a bidirectional circular audio feedback configuration. Two microphones are connected to two of the eight delay lines, feeding the network with the sound from the piano, and each node's output is connected to a loudspeaker (see Figure 1). The nodes have an independent time-varying delay. These nodes also contain sound transformations like resonators and ring modulators. Apart from the technical implementation, the behavior of the system provides a practical example of how sonic interactions may occur. The network acts as a Larsen effect that is triggered and perturbed by the sound from the piano. Although the system design is relatively simple, a high number of loops and sub-loops are activated between microphones and loudspeakers. As a result, the output of each node is dependent on the signal from the piano and from all of the other nodes, in turn feeding back to the network. It thus becomes possible to hear the 
Figure 1. Burn's setup for Cage's Electronic Music for Piano.

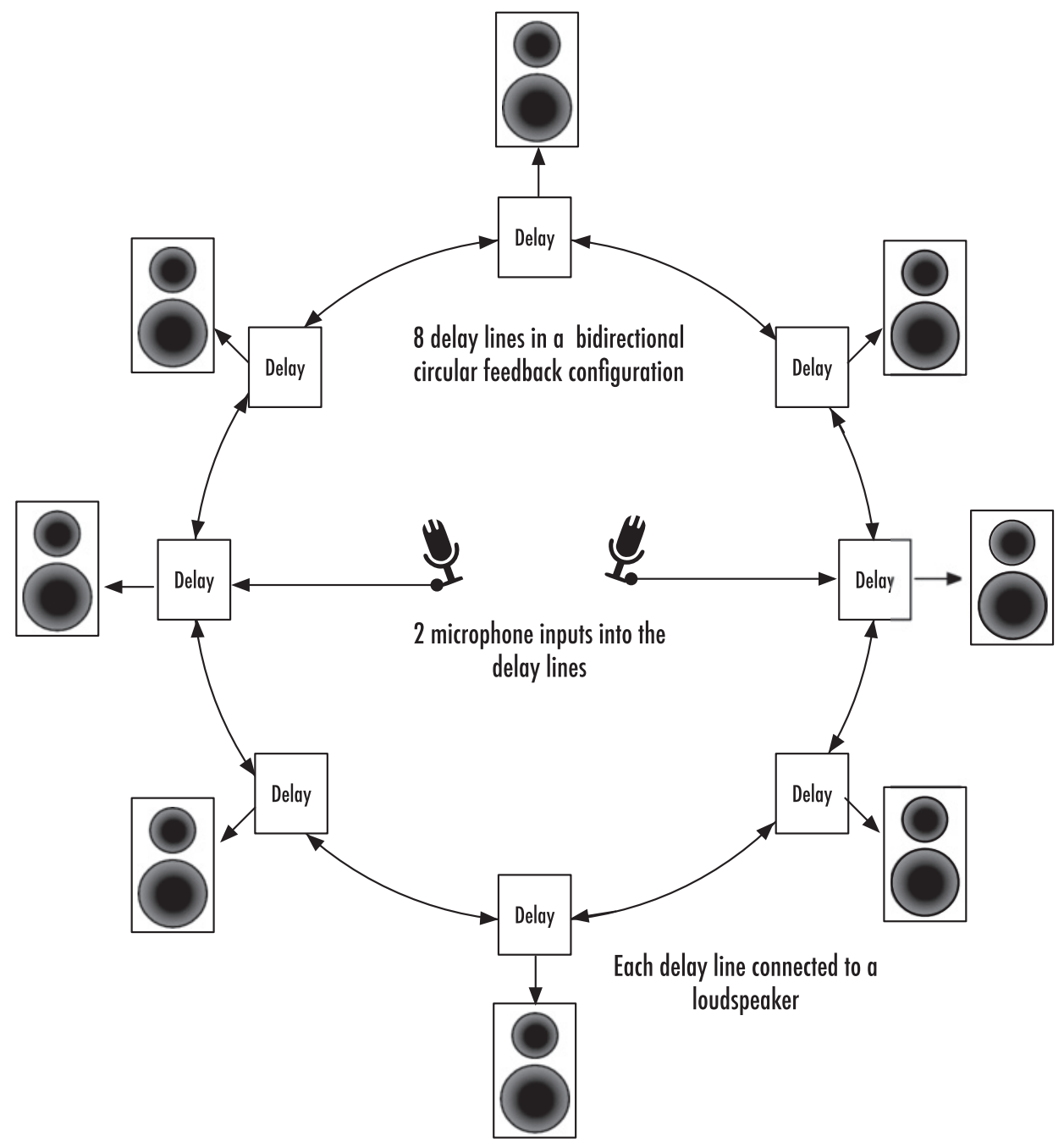

sound of the single nodes together with their mutual influence. (A new realization of this work by Cagerecorded by Di Scipio and Ciro Longobardi in 2011 and available on Stradivarius, STR 33927-attests to its relevance to the topic of feedback systems.)

\section{Self-(Dis)organization, Homeorhesis, and Homeostasis}

Self-organization has received many definitions in different contexts, including cybernetics, information theory, thermodynamics, synergetics, and others; and although the term is widely used, there is no generally accepted meaning (Gershenson, Heylighen, and Apostel 2003).

Here we will describe the main features of selforganization so that it becomes possible to apply the concept to the musical domain as a property that characterizes feedback systems. Intuitively, self-organization happens when a system is capable of organizing itself autonomously, without an external entity (Ashby 1947, 1962). According to this definition, any automated music system might be considered self-organizing. In order to provide a stricter definition, self-organization can be defined 
as the emergence of coherent patterns at a global level out of local interactions between the elements of a system (Heylighen 2001; Gershenson 2007). Because of the recursive relations between the system's components, the self-organization process is parallel and distributed (Heylighen 2001). It takes place through the simultaneous action of all the elements, none of them playing the role of a coordinator. Self-organization, indeed, is opposed to the hypothesis of a centralized control: It excludes the presence of an external element regulating the system. From this perspective, music systems in which the elements are independent (with no interaction among them, as often happens), and in which high-level processes of sound organization are automated, cannot be considered as self-organized. If the state of a system is any configuration of its variables (i.e., its overall output), self-organization can be thought of as the shift from one state to another, including the different behaviors that appear from the process of state-shifting itself. A system can enter a stable state in which the behavior either is static or exhibits a dynamic equilibrium. The opposite situation is one where there is a dynamic unstable behavior, in which the system continuously shifts from one state to another. Such a distinction opposes self-organization (stability or dynamic equilibrium)-leading to an increase in order, that is, a decrease in statistical entropy (Heylighen 2001; Heylighen and Joslyn 2001) - to self-disorganization (dynamic instability), leading to an increase in disorder.

Self-organization and self-disorganization are in some respect analogous to homeostasis and homeorhesis, the two terms indicating, respectively, a tendency towards stability and a tendency towards a certain point (an "attractor") while the system shifts through different states (following a "trajectory").

An example of a musical work that is based on the concept of self-organization is Ephemeron by Phivos-Angelos Kollias. The composer describes the system metaphorically as a living organism, its cells being sonic units in the process of adapting to the surrounding audio environment. Adaptation occurs thanks to the capability of the cells to sense the loudness features from the environment (Kollias 2008). The system is initially fed with the sound captured from the audience's applause for the piece performed just before Ephemeron. Each cell's perception of the applause is used to dynamically control the signal processing inside that cell. The work emerges from the self-organization of a mass of cells interacting mutually and with the environment, which then evolves through the time.

\section{Chaos, Complexity, and Emergence}

Chaos is a widely known term that is often used as a synonym for "unpredictability," yet the two terms do not semantically coincide. Though chaos implies unpredictability, the reverse relation is not always true: Unpredictability does not necessarily imply chaos. For example, some systems use random number generators to drive other processes. Such processes themselves are neither interrelated nor self-related, and they have no memory of themselves-randomness and process act as two separate domains. In chaotic processes, instead, what happens now is an effect caused by what happened before.

More generally, chaos can be thought of as highly dynamic behavior where order and disorder coexist and "compete," and where a causal connection between past, present, and future is established. Feedback can be modeled as a nonlinear iterated process, a formalism usually associated with mathematical models of chaotic systems (Kellert 2008). In feedback systems, chaotic behavior can occur at two different levels. In situations where there is a dynamic equilibrium, although there is an overall stability, the inner activity can be highly chaotic. On the other hand, if considering homeorhesis, each of the states that the system passes through can be chaotic, though exactly within the trajectories that the system follows.

Complexity is yet another important concept that can be used to characterize feedback systems (Kellert 2008). The paradigm of complexity states that a mass of very simple processes can achieve complex and unexpected results. Indeed, feedback is an interesting case of a simple behavior that leads to unexpected results (due to nonlinearity) through 
Figure 2. A general schema

for feedback-based

audio/music systems.

iteration. In this sense, it can be described in the framework of complexity.

The notion of complexity is strictly related to that of emergence, the first defining the structural organization of the process, the second defining the quality of unexpectedness of the results. Emergence can refer to organizational levels (Lewes 1879), to self-organization (Varela, Thompson, and Rosch 1991), to entropy variation (Kauffman 1990), to nonlinearity (Langton 1990), or exclusively to complexity (Cariani 1991; Kampis 1991; Bonabeau, Dessalles, and Grumbach 1995a,b) or synergy (Corning 2002). It is no coincidence that some classic examples discussed in those fields involve auditory feedback.

In his work on synchronization, Strogatz (2003) discusses the pioneering study by Walker (1969) on cricket chirping. Groups of crickets synchronize their chirping exclusively by means of an auditory feedback mechanism, where each cricket reacts to its neighbors. Synchronized crickets thus achieve sexual advantage over isolated competitors. But complex dynamic patterns based on auditory feedback also emerge in other biological and cultural domains: A relevant example is hand clapping at concert houses, showing synchronous and asynchronous phases, again exclusively depending on auditory feedback, as each spectator (like crickets) tunes their clapping period with his or her neighbors (Néda et al. 2000).

In the previous two examples, emergence refers to organization, a level that seems to particularly fit the musical domain. An analogy can be traced between low and high organizational levels and, respectively, microstructure and macrostructure. According to this approach, a phenomenon is emergent when it manifests itself at a level L-hi as the result of components and processes taking place at a level L-lo (Bonabeau and Dessalles 1997). In feedback systems, the output of the system at the higher level (the overall sound output) results from the processes defined at a lower level, as the composer or performer exclusively focuses on "composing the interactions" (Di Scipio 2003). From a qualitative and holistic point of view, emergence is the rise of global properties coming from the interactions of lower level components, where the

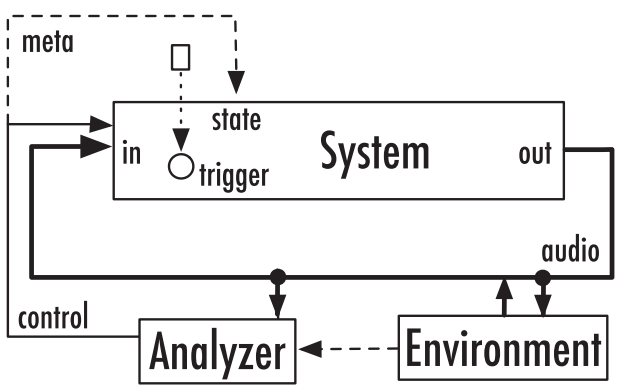

global properties are not related to those of the components (Mitchell 2006). In these cases, the synergy between the interacting components gives birth to an entity which is different from the sum of its parts (Corning 2002). It is more, but it is less, too (Morin 2006). Many important works by Di Scipio, which will be further described later, are particularly relevant in relation to the features described in this subsection, as his approach often aims at composing dynamic and chaotic entities where homeorhesis and homeostasis are competing criteria (Di Scipio 2003), and where sound and structures emerge from the sonic interactions in the environment.

\section{Towards a Typology of Music Feedback Systems}

The previous discussion, though very general, lets us narrow the field to feedback systems that are used in a wide body of musical projects dating from the 1960s and still flourishing in the present day. Although bound together by the use of feedback as a common denominator, these works present a rich and complex phenomenology which requires, in order to be fully understood, a greater articulation. First of all, it is possible to propose a general schema of audio feedback systems that aims at summing up all the key features emerging in our analysis corpus. Figure 2 shows a feedback system (System): Following the previously introduced minimal definition of feedback, System's audio output (audio, resulting from Out) is re-injected into the same system input (In). Starting from Figure 2, it is possible to propose five main features to classify feedback-based audio and music systems. These features can be organized into several oppositions, defining six categories. 
The other elements represented in Figure 2 will be introduced while discussing the categories.

\section{Information Encoding: Analog/Digital}

As an overall schema of feedback-based audio systems, Figure 2 is abstracted from the way in which information is encoded into System. Thus, a first distinction that can be taken into account is that between systems using analog encoding of information versus those that encode information digitally, although some systems may make use of both types.

A classic example of audio feedback in the analog domain can be found in Steve Reich's Pendulum Music, composed in 1968 (Reich 2002). This one-page score for microphones, loudspeakers, and performers asks for three or more microphones hanging from stands by their cables. Each stand is placed in front of a loudspeaker reproducing the signal from the corresponding microphone. The microphones are pushed back and held by the performers who will then release them simultaneously, thus making them oscillate like pendulums in front of the loudspeakers. The oscillation generates Larsen tones when the microphones are close to the loudspeakers. Cables have different lengths, meaning that both their oscillating frequency over the loudspeakers and the periods of the audio feedback loops will be different, resulting in a varying number of Larsen tones per second, and different tone pitches for each microphone-loudspeaker pair. The performance is considered finished when the microphones are still, generating continuous sounds.

Another example of a purely analog audio system is David Tudor's Microphone, commissioned by the Mills College Electronic Music Center in 1973 but originating from Tudor's work for the Pepsi Pavilion at the Expo '70 in Osaka. Tudor then tried to generalize the design to adapt it to different productions. Tudor recalled:

In the end, the number of loudspeakers in the space was 37 and there were eight input channels which could contain modifying equipment. Each of the eight input channels could have a program card which routed them to the 37 loudspeakers in different ways so that one could make circles and squares.... Now, the modifying equipment gave me gating possibilities, since by simply pointing the microphones in space and then having the sound moving between the loudspeakers at certain speeds, the feedback would occur only for an instance. There were marvelous sounds made that reminded me of being on a lonely beach, listening to birds flying around in the air (Hultberg 1988; see also Fullemann 1984).

Tudor continued, regarding the feature of the systems' self-organization, "It was wonderful. It practically did it itself."

A more recent remarkable example of a purely analog feedback system is that of the Japanese improviser Toshimaru Nakamura (www.japanimprov.com/tnakamura). Nakamura, with his No-Input Mixing Board project, creates music just by plugging the outputs of a mixer into its inputs, in this way turning a mixer into a bank of oscillators. The only tools on which he relies are amplification/attenuation of signals and equalization. The results can be surprisingly complex and interesting.

An example of a purely digital feedback system is Fantasia on a Single Number by Stelios Manousakis (online at modularbrains.net), where a single number recirculates in the feedback loops that sets the system into motion, generating sounds and structures. Other examples of digital feedback systems are the LIES (topology) performance, a work for human-machine interaction, and the $S D / O S$ (dirac) installation by Dario Sanfilippo (dariosanfilippo.tumblr.com). The two aforementioned works can be in a purely digital version, while the other LIES and SD/OS projects are basically hybrid systems. These systems are implemented by means of audio feedback networks of non-random and non-automated DSP modules like reverb, ring modulation, frequency shifting, and waveshaping, a design explicitly thought of as technically incapable of unpredictability and dynamism, yet the results are organic and evolving sonic entities with chaotic behaviors and emergent properties. 
The work by the Australian sound artist Malcolm Riddoch (online at malcolmriddoch.com), on the other hand, is an example where both digital and analog devices are used. His approach is based on improvisation, with an important focus on environmental factors, soundscape transformations, and indeterminacy. Riddoch implements hybrid feedback systems using different types of input and output transducers (microphones, electric bass, guitar), as well as analog and digital modules for sound manipulation, even using the computer to turn sound into control signals. In this way, the artist creates feedback chains aimed at exploring the spaces where the performance takes place.

\section{Information Rate: Audio/Control}

The output of an audio feedback system is an audio stream. Audio information rate can be described in relation to perception (e.g., in terms of temporal resolution of hearing; [Moore 2008]); or technology (e.g., in terms of the sampling rate in a digital system). Feedback can indeed take place in the audio domain, as happens in Larsen tones, where acoustic information from a loudspeaker is captured by a microphone, diffused again through the loudspeaker, and so on. But the rate of the signal fed back into System's input can be sub-audio, that is, occurring in the control domain, when, for example, information is extracted from sound and is used to drive processes of sound transformation.

In Figure 2 the thick line (labeled "audio") represents the audio flow re-injected into System in the case of audio feedback. In the case of control feedback, however, an analysis component (Analyzer) is required in order to perform the extraction of information from audio and to generate a control flow (the line labeled "control"). The latter feeds back to System (note that dashed lines represent derivations from sources of information).

Riddoch's system represents a situation of control feedback, and so does the I. (bar dot) project, a performance setup by Dario Sanfilippo (who contributed GenES, a DSP digital feedback system) and Andrea Valle (who contributed Objectarium, an electromechanical, computer-controlled ensemble) focusing on the exploration of feedback systems in improvisation (Sanfilippo and Valle 2012a). The systems are coupled in feedback. Namely, GenES output is analyzed by Objectarium and pilots the sequencing of events for the electromechanical ensemble, while the sound from Objectarium is fed back to GenES and perturbs its behavior. To put it in Di Scipio's (2011) words, each system listens to itself through the other self.

Another example of this type comes from work by Roberto Garretón, who, in his projects Study on Feedback I and II, implements feedback systems capable of extracting features from sound (what he calls the "senses"| such as amplitude, spectral flatness, spectral centroid, and pitch tracking. These are processed in a control DSP engine (what he calls the "brain"), and are finally used to drive sound transformations like pitch shifting, granulation, and spectrum smoothing (www.robertogarreton.com).

An example of a work based on audio feedback is the classic I Am Sitting in a Room by Alvin Lucier, written in 1969. Technically, the process adopted by Lucier, that of iteratively recording his voice after the latter is acoustically shaped by the room, is nothing but a Larsen phenomenon (in this case triggered by the voice itself) stretched in time (Di Scipio 2005). The room itself acts like a filter, and after a very high number of iterations, what you expect to hear is the resonant frequency of the room, while all other frequencies have been attenuated (for an in-depth phenomenological analysis, see also LaBelle 2006).

An example of exclusively at the control level is the Rumentario Autoedule sound installation by Andrea Valle (online at vimeo.com/37148011). The work features two main components, a computer that performs sound analysis and the scheduling of events, and an electro-mechanical orchestra made of 24 acoustic generators (the "Rumentarium" [Valle 2010]). The analysis module extracts onset, pitch, and loudness from the environment, which are then used to drive the orchestra. At each detected onset, the recognized pitch, quantized to quarter tone pitch classes, selects the next mechanical sound generator that will play, where the loudness is proportional to the current used to drive motors and actuators. As the environment coincides with the orchestra itself 
scattered over a surface, the system reacts to itself. Considering that the orchestra is highly percussive, the pitch detection is intrinsically very noisy, resulting in a dynamic and ever-changing system. Indeed, hybrid, complex configurations are clearly possible and already present in historical examples.

In 1974, Nicolas Collins realized Pea Soup, a work presenting nested positive and negative feedback in the analog domain. Namely, positive feedback is achieved by means of the Larsen phenomenon, through microphones and loudspeakers, and negative feedback is created using an analog amplitude follower that traces the energy of the Larsen tones, and whose output signal is used to pilot the phase shifting of the Larsen signal itself (Waters 2007). In this way, the stronger the Larsen phenomenon, the larger the phase shift of the signal; as a result, the signal that is fed back goes further out of phase, thus dampening the Larsen tone. This is an example of a music system in the analog domain with both audio and control feedback mechanisms, a particularly interesting one as it is probably one of the first works using feedback in the control domain.

\section{Environment Openness: Closed/Open}

A crucial aspect of feedback systems lies in their relation to the surrounding environment, represented in Figure 2 by Environment. Here we define Environment as all the audio information that is external to System, i.e., not generated or controlled by System. Indeed, sources of audio information can be of very different kinds (e.g., the surrounding soundscape captured by microphones) but also an audio stream that results from playing back audio files that are provided as input to System. A third descriptive category thus deals with the openness to the environment. Closed systems do not exchange energy/information with the environment, whereas open ones are coupled in an external feedback loop with the environment.

Nakamura's work is an example of a closed feedback system, and so is the work on feedback by David Tudor (Collins 2004), implemented by interconnecting everyday analog pedal effects and handmade electronic analog circuits (examples are available online at sites.google.com/site/futureecircuits/ david-tudor-electronics). The composer called his systems "friends," as they had the possibility of expressing themselves, the role of Tudor being that of putting into effect what was already inside the systems (O'Connell 2008).

Riddoch's work, on the other hand, is clearly oriented towards open systems, just like Lucier's works I Am Sitting in a Room (previously described) and Bird and Person Dyning. In the latter work, the American composer explores the Larsen phenomenon through ear-microphones and loudspeakers, walking through the concert hall and directing his ears.

Another interesting example of open system comes from Mark Trayle's Phantom Rooms (Trayle 2011). In 2010, Trayle was invited to create and perform a piece which would somehow interconnect two different locations 200 kilometers away (in Turin and Cuneo, Italy). Two electroacoustic music ensembles in two different locations, and the spaces themselves, were linked through a bidirectional, very fast Internet connection. The performers could share not only data but also audio streams in real time coming from the microphones placed in each location. Each ensemble was also provided with the impulse response of the other ensemble's environment, to be used to filter the sound. The name of the project was Phantom Rooms, as in some sense each location was appearing acoustically in the other one. To put it in the composer's own words, "[it is] a bit like being invited into a friend's home and bringing your own room" (Trayle 2011, p. 14).

Hybrid systems in this category are common, as it is easy to establish or break feedback with the environment through, for example, microphones and loudspeakers. The crucial point for open systems, however, is that some kind of interaction has to take place between the system and the environment, and the sole physical connection may not be enough.

\section{Trigger Modality: Internal/External}

In positive feedback systems, the initial conditions are particularly relevant, as some energy is required 
Figure 3. Overall

conceptual design of the

Audible Ecosystems

project.

in order to trigger the amplifying feedback loop. As shown in Figure 2, this initialization step (trigger, to use computing parlance) can result from the internal activity of the system itself, or it can be operated by some external agent.

In relation to the first case, an analog system always has residual noise in its components that can be used as the only source of alimentation. In the digital domain, it may be possible to have numerical garbage within the software that can be used in a similar way. Otherwise, the system can, for example, be excited with an impulse and then let run with no external sources. Another possibility is that the system features some kind of external perturbation as an element to alter its spontaneous behavior. This situation could also be considered as a particular hybrid case for the audio/control category, as the external audio is used to alter the behavior of the system, which can have effects in the short or long term.

The Nakamura and Tudor examples are also no-input feedback systems, just like some versions of the LIES and SD/OS projects. Lucier's Bird and Person Dyning is an example of the opposite feature: The mechanical bird taking part in the performance acts as a perturbation agent, triggering Larsen tones in the audio setup with its twittering.

\section{Adaptivity: Adaptive/Nonadaptive}

System is able to transform itself, i.e., to change its internal state (state), as a function of its input. This is typically the case when System is coupled with Environment. In this case, it may be capable of extracting information from Environment (see the dashed meta path in Figure 2) in order to adapt its state to changing environmental conditions. This extraction is performed by analysis algorithms at the control level (Analyzer). Because it determines a change to a different state of the system and not only a variation in its actual state, it is placed at a higher level (hence the name meta).

An example of using adaptive systems is the Audible Ecosystems project, a remarkable set of works by Agostino Di Scipio (xoomer.virgilio.it/adiscipi). The systems that belong to the project are based

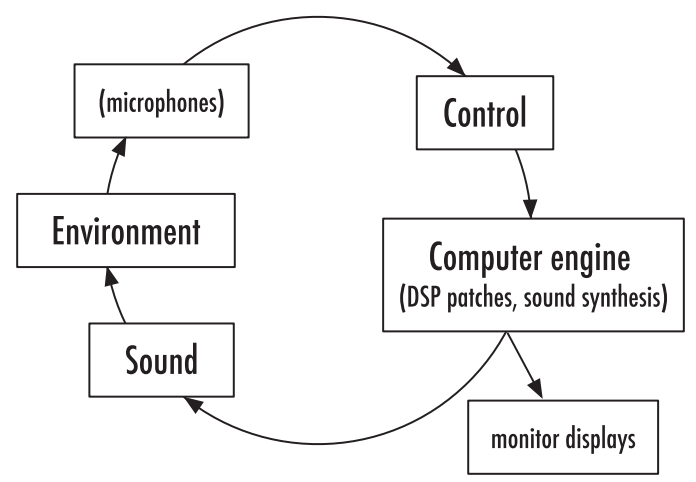

on a structural coupling with the environment, with microphones and loudspeakers being terminals through which the system exchanges energy and information with the environment. The sound is analyzed with a feature extraction algorithm: The control signal thus obtained is used to drive digital processes of sound transformation based on psychoacoustic criteria. The resulting audio is reintroduced in the environment, thus captured and analyzed again, recursively. A fundamental condition for the system to "survive" (i.e., remain active) is to be coupled with the environment (see Figure 3).

Another example of adaptive feedback system comes from Alice Eldridge, who developed the Singing Homeostat, an adaptive, sonic, kinetic sculpture whose operating mechanism is based on Ross W. Ashby's electromechanical device (homeostat) built in 1940. The sculpture consists of four oscillating mechanical dials, each of them triggering a female voice sample that fluctuates in synchronization with the dials, all together creating a microtonal drone. A software model of Ashby's device was developed as an adaptive network that replicates the original behavior of the device, tending to a stable state, while the movements of people in the environment are traced through a camera and act as perturbations of the system (Eldridge 2007).

Two further considerations are possible. First, adaptation is independent from environment coupling. A system, although coupled with the environment, may not have the capability of changing its internal state in response to a changing environment. Second, although environmental coupling is indeed the classic case for adaptation, a closed 
system can adapt to itself. That is, it can change its state as a result of its output. An interesting example comes from DSP software. The Volta software by Mark of the Unicorn is a virtual instrument plug-in that turns the sound card into a voltage control interface (www.motu.com/products/software/volta). Volta generates and sends DC signals via the sound card to external voltage-controlled analog synthesizers, thus allowing the user to attain digital control of analog hardware. In turn, the audio signal output by the analog device can be connected in a feedback loop to the sound card input and received by Volta. Through this feedback loop, Volta allows analog synthesizers to auto-calibrate. Note that, Volta is also a "hybrid control system" (Roads 1996) that couples an analog and a digital component.

\section{Human-Machine Interaction: Absent/Present}

A final category, this time exclusively musical, concerns the presence or absence of a human performer interacting with the systems and entering the feedback loop. Being structurally sensitive to minimal variations in their input, feedback systems tend to prompt an opposite performing situation. In the first case, the performer is absent, and the system is entirely machine-based. In the second case, the performer is present: Because he or she is forced to dynamically interact with the dynamic system in the design of the performance, an improvisational mood is often preferred to a fixed set of instructions. Also, as improvisation is a process where actions are causally related to listening, an aural feedback loop is established between the machine and the performer, the latter becoming an integral part of the overall system.

As feedback systems, and more generally electroacoustic and computational devices, can operate without external control or actions by the performer, the latter, while improvising, is in front of an entity that can be autonomous. In this case, the human-machine relation is not necessarily based on subordination, but rather on a nonhierarchical exchange between two entities with their own aesthetics (Rowe 1999; Garnett 2001; Bowers 2002; Sanfilippo and Valle 2012a). These features had already emerged in some of the very first computational interactive systems from the beginning of the 1970s-Joel Chadabe's CEMS and Salvatore Martirano's SalMar Construction. As noted by Chadabe, in those cases interaction indicated that "performer and instrument were mutually influential... distinctions fade between instrument and music, composer and performer. The instrument is the music" (Chadabe 1996, p. 291). Chadabe's conclusion applies indeed more generally to feedback systems. In this sense, Martirano's words, related to his now 40-year-old SalMar Construction, are enlightening:

It was too complex to analyze. But it was possible to predict what sound would result, and this caused me to lightly touch or slam a switch as if this had an effect. Control was an illusion. But I was in the loop. I was trading swaps with the logic. I enabled paths. Or better, I steered. It was like driving a bus (Chadabe 1996, p. 291).

The performer is not explicitly represented in the general schema of Figure 2 because he or she can play different functional roles. The performer can trigger the system (acting like trigger); contribute to perturbing the environment by producing or modifying sounds (that is, becoming a part of Environment); and extract information from audio (like Analyzer) in order to vary system parameters (as in the control signal flow) or to make the system change its state (like the meta flow). As the performer is theoretically a black box, the analytical treatment of his or her behavior, with respect to the other components of the feedback configuration, may be very complex and lead to ambiguous findings.

\section{Classification and Types}

Starting from the categories discussed here, it is possible to describe feedback systems by encoding the values that each system assumes for each category. In a significant number of cases it is not easy to define the value for the categories. On the one hand, the system may appear ambiguous to the 


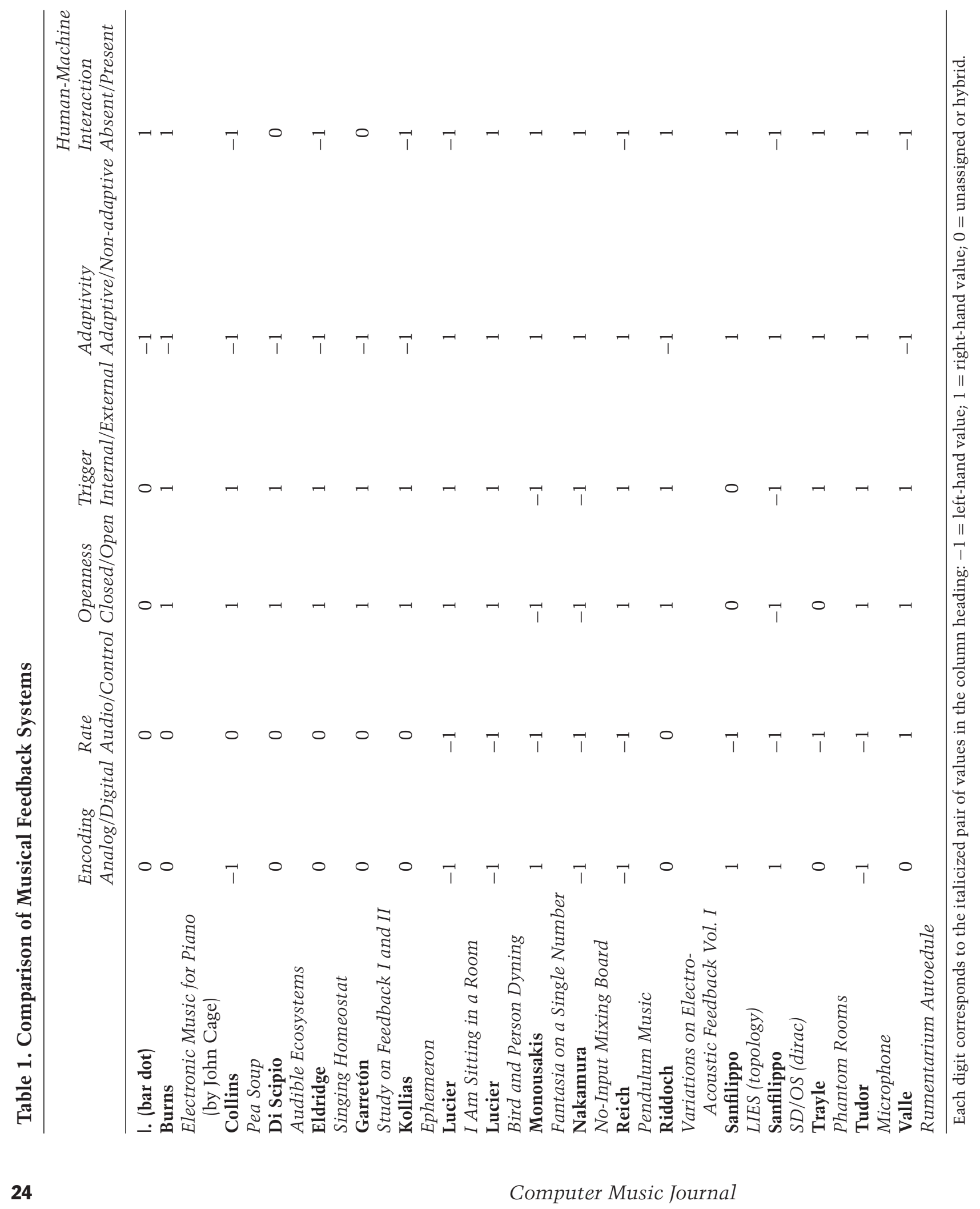


observer because of its complexity or of the lack of information on its internal processes. On the other hand, hybrid configurations are indeed possible that do not clearly allow one to place the system with respect to the category (the typical case being that of mixed analog and digital configurations).

In order to classify feedback systems, each category can receive a value of $-1,0$, or 1 where -1 and 1 represents the opposed features, and 0 the case of unassigned, hybrid systems for that category. In short, each feedback system can be represented by a ternary string encoding its properties. Table 1 shows a comparison of the previously discussed examples. Columns represent categories, rows show values for each example. As there are six categories to be taken into account, each one with three possible values, the total number of possible combinations is very high, resulting in $3^{6}=729$ types of feedback system. As a consequence, such an analytical, even if minimal, framework allows one to include many different works, sharing a common reference to feedback but coming from different traditions and practices, and to specify their mutual relations.

\section{Conclusion}

The use of feedback clearly identifies a specific group of works that, starting from the 1960s, have explored (with varying degrees of awareness) a tightly related set of notions such as nonlinearity, circular causality, interdependency, self-organization, complexity, and emergence. Even if the external boundaries that define this body of work are, if not clear-cut, at least sufficiently evident, feedback-based musical systems still show a wide internal variety that must be tackled in order to shed light on their richness. The discussed typology, resulting from the set of six general categories, is intended to provide an analytical tool that would allow description and comparison among feedback-based audio and musical works. The categories can be further expanded. As an example, interaction includes many different performing modalities that can lead to other subcategories. On the other hand, there is indeed a trade-off between analytical detail and overall manageability. Our future work will focus on expanding the body of examples in order to test the typological device and eventually modify the schema and redefine or refine the categories.

\section{Acknowledgments}

This article is a revised and expanded version of an earlier conference paper, see Sanfilippo and Valle (2012b). The authors wish to thank Agostino Di Scipio, Stefano Bassanese, Marco Cecotto, Giacomo Albert, Joshua Parmenter, and Doug Keislar for their insightful comments.

\section{References}

Ashby, W. R. 1947. "Principles of the Self-Organizing Dynamic System." Journal of General Psychology 37:125-128.

Ashby, W. R. 1956. An Introduction to Cybernetics. London: Chapman and Hall.

Ashby, W. R. 1962. "Principles of the Self-Organizing Dynamic System." In Transactions of the University of Illinois Symposium on Principles of Self-Organization, pp. 225-278.

Bonabeau, E., and J.-L. Dessalles. 1997. "Detection and Emergence." Intellectica 25:85-94.

Bonabeau, E., J.-L. Dessalles, and A. Grumbach. 1995a. "Characterizing Emergent Phenomena (1): A Critical Review." Revue Internationale de Systémique 9(3):327346.

Bonabeau, E., J.-L. Dessalles, and A. Grumbach. 1995b. "Characterizing Emergent Phenomena (2): A Conceptual Framework." Revue Internationale de Systémique 9(3):347-369.

Boner, C. P., and C. R. Boner. 1966. "Behavior of Sound System Response Immediately Below Feedback." Journal of the Audio Engineering Society 14(3):200203.

Bowers, J. 2002. "Improvising Machines. Ethnographically Informed Design For Improvised Electro-Acoustic Music." Master's thesis, KTH Royal Institute of Technology, Stockholm.

Burns, C. 2004. "Designing for Emergent Behavior: A John Cage Realization." In Proceedings of the International Computer Music Conference, pp. 193-196.

Cariani, P. 1991. "Adaptivity and Emergence in Organisms and Devices." World Futures 31:49-70. 
Chadabe, J. 1996. Electric Sound. The Past and Promise of Electronic Music. Upple Saddle River, New Jersey: Prentice Hall.

Collins, N. 2004. "Composers Inside Electronics: Music After David Tudor." Leonardo Electronic Almanac 14.

Cook, P. 1992. "A Meta-Wind-Instrument Physical Model and a Meta-Controller for Real Time Performance Control." In Proceedings of the International Computer Music Conference, pp. 273-276.

Corning, P. 2002. "The Re-emergence of Emergence: a Venerable Concept in Search of a Theory." Complexity 7(6):18-30.

de Rosnay, J. 1997. "Feedback. Principia Cybernetica Web." Available online at pespmc1.vub.ac.be/ FEEDBACK.html. Accessed 16 February 2012.

Di Scipio, A. 2003. "Sound Is the Interface: From Interactive to Ecosystemic Signal Processing." Organised Sound 8(3):269-277.

Di Scipio, A. 2005. "Per una crisi dell'elettronica dal vivo (analisi del brano di Lucier)." Rivista di Analisi e Teoria Musicale XI(2):111-134.

Di Scipio, A. 2011. "Listening to Yourself through the Otherself: On Background Noise Study and Other Works." Organised Sound 16(2):97-108.

Eldridge, A. 2007. "Collaborating with the Behaving Machine: Simple Adaptive Systems for Generative and Interactive Music." Ph.D. thesis, School of Cognitive Science, University of Sussex.

Fullemann, J. D. 1984. "“...Performing Is Very Much Like Cooking: Putting It All Together, Raising.' An Interview with David Tudor." Available online at davidtudor.org/Articles/fullemann.html. Accessed November 2012.

Garnett, G. E. 2001. "The Aesthetics of Interactive Computer Music." Computer Music Journal 25(1):2133.

Gershenson, C. 2007. "Design and Control of Selforganizing Systems." Ph.D. thesis, Faculteit Wetenschappen and Center Leo Apostel for Interdisciplinary Studies, University of Brussels.

Gershenson, C., F. Heylighen, and C. L. Apostel. 2003. "When Can We Call a System Self-organizing?" In Advances in Artificial Life: 7th European Conference. Berlin: Springer, pp. 606-614.

Ghazala, R. 2005. Circuit-Bending. Build Your Own Alien Instruments. Indianapolis, Indiana: Wiley.

Heylighen, F. 2001. "The Science of Self-Organization and Adaptivity." The Encyclopedia of Life Support Systems 5(3):253-280.

Heylighen, F., and C. Joslyn. 2001. "Cybernetics and Second-Order Cybernetics." In R. A. Meyers, ed.
Encyclopedia of Physical Science and Technology. New York: Academic Press, pp. 155-170.

Hodgson, J. 2010. Understanding Records. A Field Guide to Recording Practice. New York: Continuum.

Hultberg, T. 1988. "I Smile When the Sound Is Singing through the Space: An Interview with David Tudor." Available online at davidtudor.org/ Articles/hultberg.html. Accessed November 2012.

Kampis, G. 1991. Self-Modifying Systems in Biology and Cognitive Science. New York: Pergamon.

Karplus, K., and A. Strong. 1983. "Digital Synthesis of Plucked-String and Drum Timbres." Computer Music Journal 7(2):43-55.

Kauffman, S. A. 1990. "Requirements for Evolvability in Complex Systems: Orderly Dynamics and Frozen Components." In Proceedings of the Ninth Annual International Conference of the Center for Nonlinear Studies on Self-organizing, Collective, and Cooperative Phenomena in Natural and Artificial Computing Networks on Emergent Computation, pp. 135-152.

Kellert, S. H. 2008. Borrowed Knowledge: Chaos Theory and the Challenge of Learning across Disciplines. Chicago: University of Chicago Press.

Kollias, P. A. 2008. "Ephemeron: Control over SelfOrganised Music." In Proceedings of the Fifth Sound and Music Computing Conference, pp. 138146.

LaBelle, B. 2006. Background Noise: Perspectives on Sound Art. London: Continuum.

Langton, C. G. 1990. "Computation at the Edge of Chaos: Phase Transitions and Emergent Computation." Physica D: Nonlinear Phenomena 42:12-37.

Lewes, G. H. 1879. Problems of Life and Mind. Cambridge, Massachusetts: Riverside Press.

Mitchell, M. 2006. "Complex Systems: Network Thinking." Artificial Intelligence 170:1194-1212.

Moore, B. C. 2008. An Introduction to the Psychology of Hearing. 5th ed. Bingley, UK: Emerald.

Morin, E. 2006. "Restricted Complexity, General Complexity." Carlos Gershenson, trans. Presented at the Colloquium "Intelligence de la complexité: épistémologie et pragmatique," Cerisy-La-Salle, France, 26 June 2005. Available online at cogprints.org/5217. Accessed November 2012.

Néda, Z., et al. 2000. "Physics of the Rhythmic Applause." Physical Revue E 61:6987-6992.

O'Connell, B. C. 2008. "Electronic Friends: David Tudor and Live Electronic Music." Master's thesis, Wesleyan University. Available online at wesscholar .wesleyan.edu/etd_hon_theses/124. Accessed November 2012. 
Patteson, T. W. 2012. "The Time of Roland Kayn's Cybernetic Music." Travelling Time, Sonic Acts XIV. Amsterdam: Sonic Acts Press, pp. 47-67.

Prieberg, F. K. 1960. Musica ex machina. Über das Verhältnis von Musik und Technik. Berlin: Ullstein.

Reich, S. 2002. Writings on Music, 1965-2000. New York: Oxford University Press.

Roads, C. 1996. The Computer Music Tutorial. Cambridge, Massachusetts: MIT Press.

Rocchesso, D., and J. O. Smith. 1997. "Circulant and Elliptic Feedback Delay Networks for Artificial Reverberation." IEEE Transactions on Speech and Audio Processing 5(1):51-63.

Rowe, R. 1999. "The Aesthetics of Interactive Music Systems." Contemporary Music Review 18(3):83-87.

Sanfilippo, D., and A. Valle. 2012a. "Heterogeneouslycoupled Feedback Systems. The I. (Bar Dot) Project." In Proceedings of International Computer Music Conference, pp. 220-227.

Sanfilippo, D., and A. Valle. 2012b. "Towards a Typology of Feedback Systems." In Proceedings of International Computer Music Conference, pp. 30-37.

Scamarcio, M. 2008. "Space as an Evolution Strategy. Sketch of a Generative Ecosystemic Structure of Sound." In Proceedings of the Sound and Music Computing Conference, pp. 95-99. Available online at www.smc-conference.org/smc08/images/ proceedings/session6_number1_paper44.pdf. Accessed November 2012.

Schöffer, N. 1954. "Le Spatiodynamisme." Paper presented at the Conference of the Société Française
d'Esthétique, 19 June 1954. Available online at www.olats.org/schoffer/spatiody.htm. Accessed March 2013.

Strogatz, S. H. 2003. Sync: The Emerging Science of Spontaneous Order. New York: Hyperion.

Trayle, M. 2011. "Remote Utopias/Phantom Room." In Prossime distanze. Atti del XVIII CIM - Colloquio di Informatica Musicale, pp. 11-14.

Valle, A. 2010. "The Rumentarium Project." In Proceedings of the ACM International Conference on Multimedia, pp. 1413-1416.

Varela, F., E. T. Thompson, and E. Rosch. 1991. The Embodied Mind. Cambridge, Massachusetts: MIT Press.

Viola, B. 1995. Reasons for Knocking at an Empty House. Writings 1973-1994. London: Thames and Hudson.

Viola, B. 2004. "David Tudor: The Delicate Art of Falling." Leonardo Music Journal 14:49-56.

von Bertalanffy, L. 1968. General System Theory: Foundations, Development, Applications. New York: Braziller.

Walker, T. J. 1969. "Acoustic Synchrony: Two Mechanisms in the Snowy Tree Cricket." Science 166(3907):891894.

Waters, S. 2007. "Performance Ecosystems: Ecological Approaches to Musical Interaction." In Electroacoustic Music Studies Network. Available online at webuser.fhfurtwangen.de/friedm/PerformEcosystems.pdf. Accessed November 2012.

Wiener, N. 1948. Cybernetics; or Control and Communication in the Animal and the Machine. New York: Wiley. 